\title{
WHIPPLE'S DISEASE - CLINICAL SPECTRUM AND DIAGNOSTIC APPROACH (WITH A CONTRIBUTION OF EIGHT CASES)
}

\author{
Diana Gancheva ${ }^{1}$, Miglena Stamboliyska ${ }^{1}$, Maria Atanasova ${ }^{1}$, Iskren Kotzev ${ }^{1}$, \\ Maria Tzaneva ${ }^{2}$ \\ ${ }^{1}$ Clinic of gastroenterology, hepatology and nutrition, ${ }^{2}$ Department of general and clinical \\ pathology, St. Marina University Hospital of Varna, Medical University of Varna
}

\begin{abstract}
PURPOSE: Whipple's disease (WD) is a rare systemic infection caused by the bacterium Tropheryma whipplei. Since the clinical features of the disease are non-specific, diagnosis still remains a challenge. The aim of the study is to analyse the clinical presentation and diagnostic approach in patients with WD.

MATERIAL AND METHODS: Eight patients, six females and two males at an average age of 59 years (range, 46-79 years) were diagnosed with WD from January, 2012 to May, 2013 in the Clinic of Hepatogastroenterology, St. Marina University Hospital of Varna. Laboratory tests, endoscopic, radiologic, ultrasound and histomorphological examinations were performed.

RESULTS: The main symptoms are abdominal pain, chronic diarrhoea and meteorism. Only one female patient presents with clinical signs of malabsorbtion, such as weight loss, anasarca, ascites, pleural effusions and anaemia. There are no extraintestinal manifestations. Tests for Chlamydia trachomatis, tuberculosis and Clostridium difficile are negative. Stool examination does not show any parasitic or bacterial infection. Coeliac disease (CD) serological tests are negative, except in one female with co-existing gluten enteropathy since childhood onwards, where Crohn colitis is diagnosed, too. Endoscopy demonstrates mild to moderate atrophy of the intestinal mucosa. Histological examination establishes mild villous atrophy, lymphoplasmatic infiltration and lymph vessel dilation. All the biopsies show PAS-positive inclusions in the macrophages. Doxycycline therapy exerts a favourable effect on the clinical symptoms in all the patients.

CONCLUSION: Whipple's disease (WD) is a rare systemic disease that is commonly late or falsely diagnosed. The prognosis of non-treated patients is poor. The disease should be considered in any patients with prolonged gastrointestinal symptoms such as unexplained abdominal pain, diarrhoea and features of malabsorption syndrome. The appropriate antibiotic treatment achieves remission and improves the patients' quality of life.
\end{abstract}

Keywords: Whipple disease, diagnosis, histology, malabsorption, treatment

Address for correspondence:

Diana Gancheva, MD

Department of gastroenterology,

St. Marina university hospital of Varna,

1 Hristo Smirnenski Str., 9010 Varna, Bulgaria

e-mail:gancheva_vn@abv.bg

Received: April 5, 2014

Accepted: June 4, 2014

\section{INTRODUCTION}

Whipple's disease (WD) is a rare systemic infection caused by bacterium Tropheryma whipplei. Its main clinical manifestations include weight loss, gastrointestinal symptoms, malabsorption syndrome, anaemia, arthralgia and arthritis, lymphadenopathy, neurological manifestations, ocular affection, and endocarditis. Because of its rarity, the dis- 
Diana Gancheva, Miglena Stamboliyska, Maria Atanasova et al.

ease is, commonly, not considered within the differential diagnosis.

The objective of this communication is to describe the clinical symptoms and diagnostic approach in eight WD patients of ours.

\section{MATERIAL AND METHODS}

Eight patients, six females and two males at an average age of 59 years (range, 46-79 years) were diagnosed with WD from January, 2012 to May, 2013 in the Clinic of Hepatogastroenterology, St. Marina University Hospital of Varna. Laboratory tests, serologic tests for Chlamydia trachomatis, antigliadin and anti-tissue transglutaminase antibodies, microbiologic and parasitologic stool tests, Clostridium difficile toxin A and B were performed. Abdominal echography, intestinal passage was carried out in one patient, computed tomography (CT) colonoscopy in two, fibrogastroduodenoscopy with biopsy in seven, and fibrocolonoscopy in six patients.

\section{RESULTS}

The most common symptoms in our patients were the following: abdominal pain in seven patients and chronic diarrhoea in six ones. The number of defecations varied from two to six per 24 hours. One female patient shared the occurrence of pathological stool admixtures such as mucus and blood spots. Meteorism was established in 6 patients, weight reduction in one half of the patients, general fatigue in three patients and appetite loss in one patient. Clinical signs of a manifested malabsorption syndrome consisting of weight reduction, ascites, pleural effusions, anasarca, and anaemia (Table 1) were detected in one 74-year old female patient presenting with complaints of chronic diarrhoea. Both of the oldest female patients complained of arthralgiae which were not additionally specified. They could be related to osteoporosis, too.

Physical examination established signs of anaemia such as pale skin and mucosae along with reduced subcutaneous adipose tissue in four patients. As aforementioned, there was ascites along with lower limb oedema, anasarca and pleural effusions in one female patient. The inspection of the other organs and systems failed to reveal any other abnormalities in the rest of the patients.
Laboratory tests demonstrated normocytic anaemia in three patients and hypochromic irondeficiency anaemia in one patient. Leukocyte count was normal in all the cases. There was thrombocytosis in two cases. Elevated C-reactive protein was proved in three cases. We established hypoproteinaemia and hypoalbuminaemia in the female patient who presented with malabsorption syndrome.

Electrolytes, renal function, and liver enzymes were within normal limits in our patients. The tests for Chlamydia trachomatis, HIV, tbc and Clostridium difficile toxin $\mathrm{A}$ and $\mathrm{B}$ were negative. The stool test failed to identify any parasitic or bacterial infection. The serologic tests for gluten enteropathy (celiac disease, $\mathrm{CD}$ ) were negative in all the patients except in one female with already proven gluten enteropathy since childhood onwards.

The abdominal echography established hepatic steatosis in five patients, slight intestinal wall oedema in two, ascites, small pericardiac effusion and pleural effusions as well in the female patient with a manifested malabsorption syndrome. There was minimal ascites and small pericardiac effusion, without any evidence of hypoalbuminaemia, in one male patient presenting with abdominal pain, chronic diarrhoea and iron-deficient anaemia.

The fibrogastroscopy identified signs of gastritis in all the patients as well as a hyperplastic polyp and gastric ulcer at a cicatrisation stage in one patient each. There was a slight to moderate mucosal atrophy in the postbulbar part of the duodenum. In the aforementioned female patient, whitish spot-like mucosal swellings were described.

The histological examination established a slight villous atrophy, lymphocytic and plasmocytic inflammatory infiltration and lymph vessel dilatation. Macrophages and PAS-positive cytoplasmic inclusions were seen in all the biopsy specimens. Such a finding was detected with the oldest female patient in the gastric biopsy, too. One male patient underwent fibrocolonoscopy, only that established a slight atrophy of the terminal ileal mucosa. The typical PASpositive particles in the macrophages were histologically observed (Fig. 1, Fig. 2).

The fibrocolonoscopy revealed slight non-specific colitis, diverticulosis without diverticulitis in two patients and adenomatous polyps in one pa- 


\begin{tabular}{|c|c|c|c|c|}
\hline $\begin{array}{l}\text { Patient } \\
\text { No }\end{array}$ & Gender & $\begin{array}{l}\text { Age at diagnosis } \\
\text { (years) }\end{array}$ & Clinical symptoms & Treatment \\
\hline 1 & female & 74 & $\begin{array}{l}\text { Diarrhoea, weight loss, anasarca, } \\
\text { weakness, anorexia, normocytic } \\
\text { anaemia, hypoalbuminaemia, } \\
\text { malabsoption, arthralgia }\end{array}$ & $\begin{array}{l}\text { Doxycycline p.o. } 2 \times 100 \\
\text { mg, probiotics, Kreon }\end{array}$ \\
\hline 2 & female & 51 & $\begin{array}{l}\text { Abdominal pain, diarrhoea, } \\
\text { meteorism }\end{array}$ & $\begin{array}{l}\text { Doxycycline p.o. } 2 \times 100 \\
\text { mg, probiotics, Kreon }\end{array}$ \\
\hline 3 & female & 63 & $\begin{array}{l}\text { Abdominal pain, diarrhoea, weight } \\
\text { loss, normocytic anaemia }\end{array}$ & $\begin{array}{l}\text { Doxycycline p.o. } 2 \times 100 \\
\text { mg, probiotics, Kreon }\end{array}$ \\
\hline 4 & female & 56 & $\begin{array}{l}\text { Abdominal pain, diarrhoea, } \\
\text { meteorism }\end{array}$ & $\begin{array}{l}\text { Doxycycline p.o. } 2 \times 100 \\
\text { mg, probiotics, Kreon }\end{array}$ \\
\hline 5 & male & 46 & Abdominal pain, meteorism & $\begin{array}{l}\text { Doxycycline p.o. } 2 \times 100 \\
\text { mg, probiotics, Kreon }\end{array}$ \\
\hline 6 & male & 47 & $\begin{array}{l}\text { Abdominal pain, diarrhoea, } \\
\text { meteorism, weight loss, weakness, iron- } \\
\text { deficiency anaemia }\end{array}$ & $\begin{array}{l}\text { Doxycycline p.o. } 2 \times 100 \mathrm{mg} \text {, } \\
\text { probiotics, Kreon }\end{array}$ \\
\hline 7 & female & 55 & $\begin{array}{l}\text { Abdominal pain, meteorism, weight } \\
\text { loss, weakness, normocytic anaemia }\end{array}$ & $\begin{array}{l}\text { Doxycycline p.o. } 2 \times 100 \mathrm{mg} \text {, } \\
\text { probiotics, Kreon }\end{array}$ \\
\hline 8 & female & 79 & $\begin{array}{l}\text { Abdominal pain, diarrhoea, } \\
\text { meteorism, arthralgia }\end{array}$ & $\begin{array}{l}\text { Doxycycline p.o. } 2 \times 100 \mathrm{mg} \text {, } \\
\text { probiotics, Kreon }\end{array}$ \\
\hline
\end{tabular}

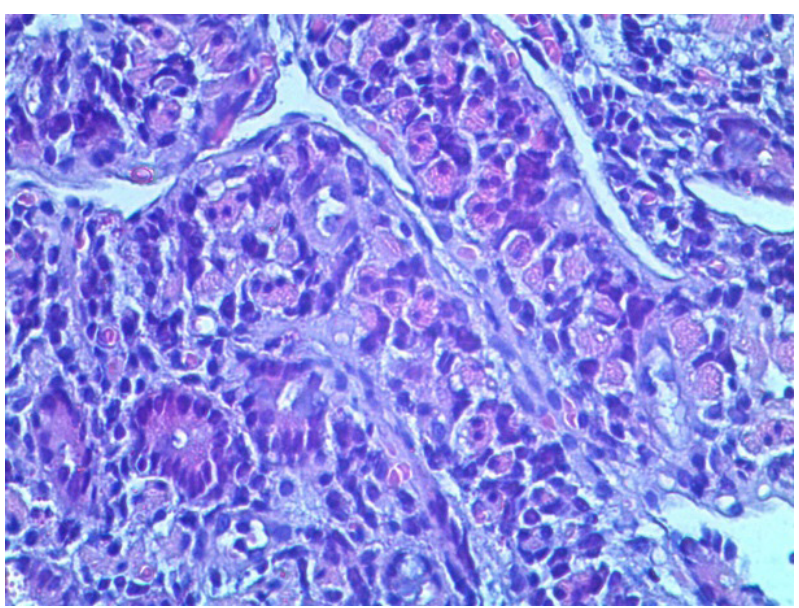

Fig. 1. Whipple's disease: foamy macrophages. Hematoxylin-eosin staining, $x 200$

tient. In the female patient with gluten enteropathy, the endocopic picture demonstrated an inflammatory bowel disease of intermediary type, more typical of Crohn's colitis, and morphological patterns resembling ulcerative colitis. The contrasted $\mathrm{x}$-ray examination of the intestine proved a delayed intestinal passage and rigid, narrowed terminal ileum. There

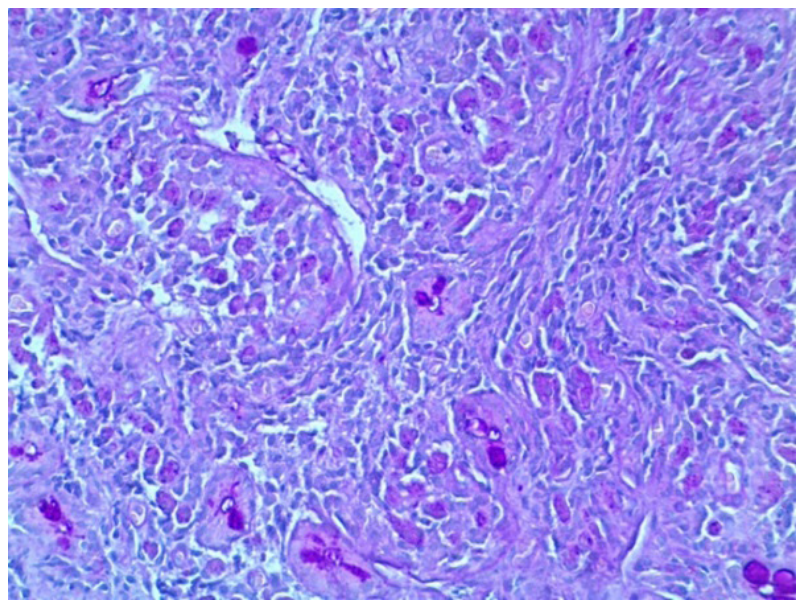

Fig. 2. Whipple's disease: PAS-positive inclusions in macrophages. PAS staining, $x 200$

were no colonoscopic abnormalities in the other two patients.

In all the patients, a treatment with doxycycline in a dose of $100 \mathrm{mg}$ twice daily in combination with probiotic and enzyme preparation, i. e. Kreon, was initiated. This resulted in favourable effects on the clinical symptoms such as reduction of the abdom- 
Diana Gancheva, Miglena Stamboliyska, Maria Atanasova et al.

inal pain and meteorism, stool normalisation, tendency of body weight normalisation and general status improvement.

\section{DISCUSSION}

WD was first described in 1907 by the American pathologist George Hoyt Whipple. The infectious etiology was suspected already in the first communication where he described numerous rod-like organisms in the vacuoles of the foamy cells. In 1991, the microorganism was identified by polymerasechain reaction (PCR) and named Tropheryma whipplei. It was first cultivated in 2000 . WD is rare. Until present days, there are about 1000 case reports of this disease $(2,3,7,12,14)$. Most commonly, Caucasian males at an average age of 50 years are affected. In contrast, in our contingent, the female gender prevails. The epidemiological investigations based on a comparative analysis for the last decades of the XX century demonstrate a significant increase of the relative share of the females affected by the disease (2). A chronic asymptomatic carriership was proved through examination of the saliva and fecal samples from healthy individuals $(3,7,13)$. A feco-oral, orooral and air-drop infection transmission route was suggested $(8,9)$.

The pathogenesis of the disease remains not clarified yet. One describes a reduced or even absent specific Th1-cell response along with a deficit of the macrophageal function disturbing the degradation of the bacterial antigens. That is why it is assumed that the underlying immune deficiency of the organism is responsible for WD development $(3,5-7,14)$. The combination of three comparatively rare diseases in the same female patient of ours, such as gluten enteropathy, WD and Crohn's disease is an interesting fact that confirms the conception of the role of the immunological factors in their pathogenesis.

The classic disease is characterised by a prodromal stage with non-specific symptoms, most often arthralgia and non-deforming migrating arthritis which is one of the most frequent manifestations of the disease. Next follows the stage of the gastrointestinal symptoms such as diarrhoea, weight loss and weakness, and last comes the generalised stage consisting of anaemia, steatorrhoea, fever, polyserositis (including pericarditis, pleural effusions and ascites), hypoalbuminaemia, malabsorption as well as neurological and/or cardiovascular manifestations $(3,13)$.

Only the two oldest female patients complained of arthralgia which could, probably, be due to osteoporosis, too. The gastrointestinal symptoms occurred in any patients of ours. Two patients presented with pleural effusions, pericarditis, and ascites. These effusions could, probably, represent a manifestation of infection-related polyserositis. In the female patient with an outlined malabsorption syndrome, the pleural effusion was of inflammatory nature which suggested the accompanying involvement of an infectious agent in the pathogenesis of the exudate.

The neurological manifestations can resemble almost any neurological conditions $(3,7,13)$. There are cognitive and psychotic abnormalities. Certain ocular events such as disorders of ocular muscle movements, glaucoma, keratitis, uveitis, retinitis, etc. are described, too $(3,5,7,14)$. Heart damage presents with pericarditis, heart failure, and culture-negative endocarditis (3-5,7,12). In some patients, there are pulmonary manifestations such as pleural effusions, interstitial lung disease, pulmonary hypertension, and lung nodules $(7,12,15,16)$. It has been established that bacteriemia along with fever can be a usual clinical manifestation of an acute infection with Tropheryma whipplei (8). It has been concluded that this microorganism is widely disseminated and thus a reason for the underestimated clinical manifestations, respiratory infections inclusive $(8,9)$. Hyperpigmentations as skin patterns are seldom observed $(3,8)$.

None of our patients presented with neurological symptoms similarly to recently reported cases $(3,13)$ or other extraintestinal manifestations. Kidneys are rarely affected. A secondary amyloidosis with a nephrotic syndrome as well as IgA nephropathy occurring several years prior to the abdominal symptoms are also reported $(7,11)$. There is scant clinical data about WD of the appendix, with normal histology of the duodenal biopsy specimen (10), eosinophilic vasculitis (1), damage of the esophagus, colon, hypothalamic and renal function, without any malabsorption (3).

The upper gastrointestinal endoscopy with duodenal biopsy is the diagnostic method of choice. One describes reduced mucosal folds with uneven and rough surface and numerous small whitish-yel- 
lowish fields and plaques which alternate with an eroded, erythematous or easily vulnerable mucosa in the postbulbar area $(2,3,7)$. The histological examination of the intestinal material reveals foamy macrophages containing PAS-positive cytoplasmic inclusions, large lipid drops in lamina propria of the flat damaged intestinal villi as well as dilated lymph nodes (13). We established similar endoscopic and morphological alterations in our patients. PAS-positive macrophages could infiltrate numerous other organs (2).

The non-caseating epithelial cell or giant cell granulomas occur in some patients, most commonly, in the abdominal lymph nodes. Sarcoma-like granulomas can be found in numerous organs of WD patients. That is why this disease is an important consideration in the patients with idiopathic granulomas even in the absence of typical symptoms $(7,11)$.

The immunohistochemical examination, PCR techniques, culture test for Tropheryma whipplei and electron microscopy are sensitive diagnostic methods, however, they are not available for routine use yet.

The differential diagnosis of WD is broad and covers any diseases presenting with malabsorption and malnutrition $(3,14)$. It should be born in mind in a pulmonary, connective-tissue, or inflammatory intestinal disease as well as in the cases with fever of unknown origin. The diagnosis can be difficult in oligosymptomatic individuals without any gastrointestinal manifestations. It is necessary to correctly evaluate the possible psychiatric and neurological symptoms as well as the cases of hypothalamic and adrenal dysfunctions.

WD was fatal prior to the antibiotic usage for its treatment. There is no general consensus about the therapy of this disease. There is a natural resistance of Tropheryma whipplei towards the fluoroquinolones $(3,7)$. The sensitivity of the microorganism towards trimethoprim-sulfamethoxazole is due to sulfamethoxazole only.

Nowadays, the initiation of the therapy with antibiotics which pass through the blood-brain barrier is preferred in order to reduce the risk of relapses. It is recommended to use intravenous ceftriaxone in a dose of $1,0 \mathrm{~g}$ once daily or intravenous penicillin $G$ in a dose of 2-4 million units every 4 hours for
2-4 weeks followed by peroral trimethoprim-sulfamethoxazole in a dose of $960 \mathrm{mg}$ twice daily for one to two years. There is information about a successful treatment with penicillin $\mathrm{G}$ or amoxicillin-clavulanic acid plus gentamycin intravenously for two weeks, followed by a peroral doxycycline in a dose of $100 \mathrm{mg}$ daily plus salazopyrine in a dose of $3 \mathrm{~g}$ daily for one year.

All of our patients were treated with doxycycline for 6-9 months. The clinical symptoms were very favourably influenced. Human interferon gamma was successfully applied in the patients with damage of the central nervous system and in cases with a refractory disease $(3,7)$. Prior to hospitalisation, all of our patients were treated with spasmolytic means, proton pump inhibitors, enzyme preparations and probiotics without any influence on the symptoms at all. The endoscopic examination along with the duodenal biopsy and purposeful search for this disease contributed to the exact diagnosis and beginning of the appropriate antibiotic therapy.

\section{CONCLUSION}

WD is a chronic infectious systemic disease characterised by a variety of clinical manifestations and thus often with a false or late diagnosis. Its prognosis, without any treatment, remains poor. Although WD is rare, our awareness of it focused our clinical search on its detection. Our patients prove that WD should be born in mind in any cases of long-lasting gastrointestinal symptoms such as unexplained abdominal pain, diarrhoea, weight reduction, malabsorption symptoms, anaemia, independently of the age and gender, despite the absence of the whole range of classical clinical manifestations as in our own cases. The appropriate long-lasting antibiotic therapy achieves a favourable development of the clinical symptoms and durable remission of the disease.

\section{REFERENCES}

1. Al-Hamoudi, W., F. Habbab, C. Nudo, A. Nahal, K. Flegel. Eosinophilic vasculitis: a rare presentation of Whipple's disease.- Can. J. Gastroenterol., 21, 2007, No 3, 189-191.

2. Bai, J. C., R. M. Mazure, H. Vazquez, S. I. Niveloni, E. Smecuol, S. Pedreira, et al. Whipple's disease.- Clin. Gastroenterol. Hepatol., 2, 2004, No 10, 849-860. 
Diana Gancheva, Miglena Stamboliyska, Maria Atanasova et al.

3. Bureš, J., M. Kopáčová, T. Douda, J. Bártová, J. Tomš, S. Rejchrt, et al. Whipple's disease: our own experience and review of the literature.- Gastroenterol. Res. Pract., 2013; 2013: 478349. doi: $10.1155 / 2013 / 478349$.

4. Chhabria, M. A., R. J. Dalal, D. C. Desai, R. B. Deshpande. Whipple's disease.- J. Assoc. Physicians India, 58, 2010, 196-197.

5. Conly, J. M., B. L. Johnston. Rare but not so rare: the evolving spectrum of Whipple's disease.- Can. J. Infect. Dis., 12, 2001, No 3, 133-135.

6. Desnues, B., M. Ihrig, D. Raoult, J. L. Mege. Whipple's disease: a macrophage disease.- Clin. Vaccine Immunol., 13, 2006, No 2, 170-178.

7. Fenollar, F., X. Puechal, D. Raoult. Whipple's disease.- New Engl. J. Med., 356, 2007, No 1, 55-66.

8. Fenollar, F., O. Mediannikov, C. Socolovschi, H. Bassene, G. Diatta, H. Richet, et al. Tropheryma whipplei bacteremia during fever in rural West Africa.- Clin. Infect. Dis., 51, 2010, No 5, 515-521.

9. Greub, G. A new piece added to the Whipple puzzle: Tropheryma whipplei primary infection with bacteremia and cough.- Clin. Infect. Dis., 51, 2010, No 5, 522-524.

10. Hashim, H., S. Ahmed, S. Shami, I. Saeed. Whipple's disease of the appendix.- J. R. Soc. Med., 94, 2001, No 12, 643-644.
11. Kramer, L. C., T. A. J. M. Manschot, J. N. M. Barendregt, J. N. M. Barendregt, J. M. Smit. Whipple's disease: often a late diagnosis and a rare cause of nephropathy.- NDT Plus, 2, 2009, No 1, 55-58.

12. Muir-Padilla, J., J. B. Myers. Whipple disease. A case report and review of the literature.- Arch. Pathol. Lab. Med., 129, 2005, No 7, 933-936.

13. Renon, V. P., M. C. Appel-da-Silva, R. B. D’Incao, R. M. Lul, L. S. Kirschnick, B. Galperim. Whipple's disease: rare disorder and late diagnosis.- Rev. Inst. Med. Trop. Sao Paulo, 54, 2012, No 5, 293-297.

14. Schijf, L. J., M. C. Becx, P. C. de Bruin, S. G. van der Vegt. Whipple's disease: easily diagnosed, if considered.- Neth. J. Med., 66, 2008, No 9, 392-395.

15. Urbanski, G., P. Rivereau, L. Artu, F. Fenollar, D. Raoult, X. Puéchal. Whipple disease revealed by lung involvement: a case report and literature review.- Chest, 141, 2012, No 6, 1595-1598.

16. Villa, A., G. Nucera, A. Kostihova, A. Mazzola, P. Marino. Reversible pulmonary hypertension associated with Whipple's disease.- Case Rep. Pulmonol., 2012; 2012: 382460. doi: 10.1155/2012/382460. 